2. - An invariant formulation of the new maximum-minimum theory of eigenvalues, J. Math. Mech. 16 (1966) 213-218.

3. S. Goldberg, Unbounded linear operators: Theory and applications, McGrawHill, New York, 1966.

4. I. C. Gohberg and M. G. KreYn, Fundamental theorems on deficiency numbers, root numbers, and indices of linear operators, Uspehi Mat. Nauk 12 (1957), 43-188; English transl., Amer. Math. Soc. Transl. (2) 13 (1960), 185-264.

5. W. Stenger, The maximum-minimum principle for the eigenvalues of unbounded operators, Notices Amer. Math. Soc. 13 (1966), 731.

6. - On the variational principles for eigenvalues for a class of unbounded operators, J. Math. Mech. 17 (1968), 641-648.

The American University

\title{
ON AN ADDITIVE DECOMPOSITION OF FUNCTIONS OF SEVERAL COMPLEX VARIABLES
}

\author{
BY EDGAR KRAUT, STAVROS BUSENBERG AND WILLIAM HALL
}

Communicated by Maurice Heins, November 16, 1967

1. Introduction. Recent attempts (see [1] and the references in the same article) to extend the Wiener-Hopf technique for functions of a single complex variable to those of two or more complex variables have relied on a remark of Bochner's [2] that guarantees the required decomposition under suitable restrictions. Bochner's remark states that: if $f\left(z_{1}, \cdots, z_{n}\right), z_{j}=x_{j}+i y_{j}$, is analytic in a tube $T: \gamma_{i}<x_{i}<\delta_{i}$, $y_{i} \in(-\infty, \infty)$, and if $\int_{-\infty}^{\infty} \cdots \int\left|f\left(z_{1}, \cdots, z_{n}\right)\right|^{2} d y_{1} \cdots d y_{n}$ converges in $T$, then there exists in $T$ a decomposition $f=\sum_{i=1}^{2^{n}} f_{i}$, where each $f_{i}$ is analytic and bounded in an octant shaped tube $T_{i}$ containing the interior of $T$. Moreover, such a decomposition is unique up to additive constants. The uniqueness of the decomposition is not verified in [2] but reference is made to $\mathrm{H}$. Bohr's [3] corresponding result for functions of a single complex variable.

It is here shown that the uniqueness statement is false. However, the adjunction of the additional hypothesis that the $f_{i} \rightarrow 0$ when any one of the $x_{j} \rightarrow \infty$, in the tubes $T_{i}$, restores the uniqueness of the decomposition and justifies the use of the result in [2].

2. A counter-example. In the decomposition $f=\sum_{i=1}^{2 n} f_{i}, f_{1}$ is analytic and bounded in the tube $T_{1}: x_{i}>\gamma_{i}, y_{i} \in(-\infty, \infty), i=1,2$, $\cdots, n$, and $f_{2}$ is analytic and bounded in the tube $\left.T_{2}: x_{1}<\delta_{1}, x_{j}\right\rangle \gamma_{j}$, 
$y_{1} \in(-\infty, \infty), y_{j} \in(-\infty, \infty), j=2,3, \cdots, n$. Let $g\left(z_{2}, z_{3}, \cdots, z_{n}\right)$ be any function of the $(n-1)$ complex variables $z_{2}, z_{3}, \cdots, z_{n}$ such that it is analytic and bounded for $x_{j}>\gamma_{j}, y_{j} \in(-\infty, \infty), j=2,3$, $\cdots, n$. In particular $\prod_{j=2}^{n}\left(z_{j}-\gamma_{j}+\epsilon\right)^{-1}, \epsilon>0$, is such a function. Then the decomposition $f=\sum_{i=1}^{2^{n}} f_{i}^{\prime}$, where $f_{1}{ }^{\prime}=f_{1}+g, f_{2}{ }^{\prime}=f_{2}-g$, $f_{i}^{\prime}=f_{i}, i=3,4, \cdots, 2^{n}$, satisfies the conditions of Bochner's remark and yet the $f_{i}$ and $f_{i}^{\prime}$ do not simply differ by a constant.

3. Uniqueness of the decomposition. The decomposition implied in Bochner's theorem is obtainable by the use of Cauchy integrals as in the case of functions of a single complex variable [3]. However, the $f_{i}$ obtained from the Cauchy integrals when $f$ is of bounded $L_{2}$ norm in $T$ are not only bounded in $T_{i}$, but possess the asymptotic property $f_{i} \rightarrow 0$ as $x_{j} \rightarrow \pm \infty$ in the tube $T_{i}$, for any $j=1,2, \cdots, n$. It is just this asymptotic property that ensures the uniqueness of the decomposition.

THEOREM 1. Let $f\left(z_{1}, \cdots, z_{n}\right)$ be analytic in $T$, and suppose that, in $T, f=\sum_{i=1}^{2^{n}} f_{i}$, where each of the $f_{i}$ is analytic and bounded in $T_{i}$ and $f_{i} \rightarrow 0$ as any one of the $x_{j} \rightarrow \pm \infty$ in $T_{i}$. Then the decomposition is unique.

Proof. Suppose that $f=\sum_{t=1}^{2^{n}} f_{i}=\sum_{i=1}^{2^{n}} f_{i}^{\prime}$ in $T$. Then $\sum_{i=1}^{2^{n}} \Delta f^{i}$ $=\sum_{i=1}^{2^{n}}\left(f_{i}-f_{i}^{\prime}\right)=0$ in $T$. When $\gamma_{j}<x_{j}<\delta_{j}, y_{j} \in(-\infty, \infty), j=2,3$, $\cdots, n, 2^{n-1}$ of the terms in this last sum are analytic and bounded for $x_{1}>\gamma_{1}, y_{1} \in(-\infty, \infty)$, and $\rightarrow 0$ as $x_{1} \rightarrow+\infty$, while the other $2^{n-1}$ terms are analytic for $x_{1}<\delta_{1}, y_{1} \in(-\infty, \infty)$, and $\rightarrow 0$ as $x_{1} \rightarrow-\infty$. Denoting the sum of the first set by $[\Sigma \Delta f]_{+}$and of the second by $[\Sigma \Delta f]_{-}$, it follows that $[\Sigma \Delta f]_{+}=-[\Sigma \Delta f]_{-}=g\left(z_{1}, \cdots, z_{n}\right)$ in $T$. Now $g\left(z_{1}, \cdots, z_{n}\right)$ is analytic in all variables in $T$, and analytic and bounded for all $z_{1}$ whenever $\gamma_{j}<x_{j}<\delta_{j}, y_{j} \in(-\infty, \infty), j=2,3, \cdots, n$. By Liouville's theorem $g\left(z_{1}, \cdots, z_{n}\right)$ is independent of $z_{1}$, say $g\left(z_{1}, \cdots, z_{n}\right)=G\left(z_{2}, z_{3}, \cdots, z_{n}\right)$. But $\lim _{x_{1} \rightarrow \infty}[\Sigma \Delta f]_{+}=0$, hence $\lim _{x_{1} \rightarrow \infty} G\left(z_{2}, \cdots, z_{n}\right)=G\left(z_{2}, \cdots, z_{n}\right)=0$, and $[\Sigma \Delta f]_{+}=0,[\Sigma \Delta f]_{-}=0$, in $T$. Now the same argument is applied to each of the above two equations on the variable $z_{2}$, resulting in four new equations of the same form, each involving $2^{n-2}$ summands. Repeating this process for $z_{3}, z_{4}, \cdots, z_{n}$, one finds that at each step the number of homogeneous equations is doubled, while the number of summands is halved. By the $n$th step, there are $2^{n}$ equations each involving one $\Delta f_{i}$. Thus $\Delta f_{i}=0$ in $T$, and hence in $T_{i}, i=1,2, \cdots, n$, and the proof is complete.

COROLlaRY 1. Under the conditions of Bochner's theorem, $\exists f_{i}$ such 
that $f=\sum_{i=1}^{2^{n}} f_{i}$ in $T$, where the $f_{i}$ are analytic and bounded in $T_{i}$, and $f_{i} \rightarrow 0$ as $z_{j} \rightarrow \infty$, for any $j=1,2, \cdots, n$, in $T_{i}$. This decomposition is unique.

CoRollary 2. If $f\left(z_{1}, \cdots, z_{n}\right)$ is analytic and bounded in $T$, and if $f$ possesses a bounded indefinite integral $F$ in $T$ such that $f=\partial^{n} F / \partial z_{1}$ $\cdots \partial z_{n}$, then in $T, f=\sum_{i=1}^{2^{n}} f_{i}$, where $f_{i}$ is analytic and bounded in $T_{i}$, and $f_{i} \rightarrow 0$ as $x_{j} \rightarrow \pm \infty$, for any $j=1,2, \cdots, n$, in $T_{i}$. This decomposition is unique.

Proof. The existence of the decompositions postulated in the corollaries follows from the Cauchy integral theorem. The uniqueness is a consequence of Theorem 1 .

The conditions imposed on $f$ in Corollary 2 are direct extensions of the conditions that $\mathrm{H}$. Bohr [3] imposed on functions of a single complex variable.

\section{REFERENCES}

1. J. Radlow, $A$ two-dimensional singular integral equation of diffraction theory, Bull. Amer. Math. Soc. 70 (1964), 596-599.

2. S. Bochner, Bounded analytic functions in several complex variables and multiple Laplace transforms, Amer. J. Math. 59 (1937), 732-738.

3. H. Bohr, Zur Theorie der fastperiodischen Funktionen. III, Acta Math. 47 (1926), 250-251.

Science Center of North American Rockwell Corporation, Thousand OAKs, California 